you must take great pains to exclude the air, and all extraneous substances whatever, from the wound, and it is only by this method that you will ever secure union by the first intention.

Secondary hamorrhage, after eight days, came on in this case. Now secondary ha. morrhage may come on a few hours aiter an operation, after the faintness has gone off, or from the ligature having been put on carelessly. If secondary hæmorrhage do not come on in eight or ten hours, inflammation is set up, and then for a time there is no chance of hæmorrhage returning; but, after some days, ulceration com's on, and in those cases where the artery has been taken up with the needle and ligature, and the inner coat is not cut through, this ulceration produces secondary bremorrhage. It is perfectly useless for you to attempt to draw out the bleeding vessel with the tena. culum. You cannot do it; the parts are imbedded in lymph. Then what are you to do? Iou must remove all the dressings, open the stump, and expose the bleeding surface to the air, and, in general, the hremorrhage will cease. If it do not, you must make pressure higher up in the couise of the artery. This method proved successful in the present case. But even with these means the hamorrhage may occur every se. cond day, and if you do nothing the patient will die. Styptics seldom do any good. Caustic is better, but it is a barbarous remedy. The only thing you can do is, to operate for aneurism. If secondary hamorrhage should occur in this case, I shall cut down on the femoral artery, below the profunda, and tie it ; and this is generaliy the best method for stopping sacondary hxmorrhage from the lower extremity. I was once called to a case of secondary hæmorrhace from a wound of the palmar alch, and I cut down upon the humeral artery and tied it, and I really think this is a better plan than performing two operations for securing the ulnar and radial arteries.

\section{Examination of the Joint.-Remarks.}

On a dissection heing made of the joint no dead bone was discovered; I was therefore wrong in my diagnosis, but portions of the cartilage were here and there absorbed, and complete anchylosis had taken place. Now I think that the original disease was in the svnovial membrane of the joint, and extended thence to the superficial cellular texture, where it was more violent, and terminated in the effusion of pus. Similar symptoms to these occurred in a patient who had erysipelas in this hospital some years ago.

Mucous membranes suppurate more readily than sercus ones. The pus here was secreted by the synovial membrane, and the bones bore but slight marks of disease. In grown-up persons, suupuration and anchylosis (when the latter does occur) are seldom cured. Anchylosis is more common in grown-up persons than in adults.

In this case there were abscesses about the joints which did not heal. If they had healed she might have recovered; but, with an anchylosed joint, the abscesses did not heal, because they were pent up between muscles and tendons. The first object which you should have in view towards healing an abscess should be, to take care that the pus has a free exit. In the knee, the leg, and the thigh, abscesses are situated among muscles. Abscesses do not heal so readily among unhealtby parts as awong paits that ar healthy. Here the parts were unhealthy, and the abscesses did not therefore heal.

CASE OF

\section{IMPERFORATE HYMEN, WITH RETAINED MENSES,}

TREATED SUCCESSFULLY.

By John Colvax, M.D., Licen. King and Queen's College of Phys. Ireland; Physician to the Armagh Fever Mospital, \&c.

February 20th, 1831. Margaret Mr-, near Armagh, etat. 16, dark hair and eyes, and of lively habits, and remarkably healthy, in Nov. lust felt " an uneasiness and a stoppage in making water," and "a pain in her private parts;" she also felt pains in the " small of hor back and belly." This she bore for some time with. out examining into the cause of it, or telling any person, noteven her mother. A month since, January 20 th, she was "taken very ill," and has had no ease since. Sometimes the pain, a racking pain, commences in her back, and sometimes in both groins. It "meets round her back and belly," and this induces " a heart sichness"," and a cold trembling, and notwith. standing hot fomentations and foot-baths; she will be several hours before she recovers the natural heat, and when she may be "getting ease," she feels a great desire to pass water. A month ago she was led by the pain to examine herself, and found " a lump at the right side of her bellv, and at her private parts." She then informed her mother, who examined her, and found her "very strange." She got pills and pow-

* A common term for nausea among the common people in the north of Ireland. 
ders, which operated well, as physic, and nished after the operation, and did not rewhich gave her some small relief; she also turn. She continued to feel a little pain took castor oil, salts, semna, and other pur- after the operatıon, but nothing to what gatives, all of which relieved her a little. she had been accustomed to feel before. She also got a bottle of mixture, probably There was no hæmorrbage of any moment. anodyne, to ease her pain, and a plaster to The cure was completed by sponge tents, cover the loins, from the latter of which sle some trifling local applications, and apeexperienced great relief. After this she rients. I have seen her lately, and she was brought to me. I urdered her some blue is quite well, and her whole appearance pill and opium at night, and castor oil and very much improved. infs. of senna next morning; from this she had a perspiration; the medicine did not affect her bowels, yet she felt ease. She feels quite easy at present; tongue clean; pulse natural. She has had great ease since yesterday, when I first examined her, and drew off her water with a catheter, about a pint, and nearly natual. She has never menstruated.

On minute examination, a large globular tumour occupied the right umbilical and iliac regions, slightly moveable and painful on pressure. On senarating the labia, a tumour, oblong in shape, also projected slightly from between those parts, pointing towards the lower part, and of a dark purplish hue; to the touch this tumour evidently gave the feel of fluctuation. On examination per anum, an oblong tumour could be felt pressing towards the sacrum; this also gave the feel of fluctuation. From all these combined symptoms, I conceived that the menses were retained, and that probably the tumour in the iliac and umbilical regions, and in the lower part of the pelvis, was formed by the accumulated fluid. The hymen, as I supposed, being imperforate, I determined to make a slight one, it possible, by scratching with an abscess lancet through this membranous partition, and giving exit to the contained matter whatever it might be; I first drew off the urine by a catheter, and then scratched through this membrane, which was not more than a few lines in thickness, when immediately a stream of dark fluid blood rushed out to a considerable distance: it appeared like brown paint. This I allowed to flow until about three half pints came away; it then became quite thick and viscid; then $I$ en. larged the aperture upwards and down. wards to about one inch, and allowed it all to flow out, being occasionally oblined to drag out thickish grumous shreds. I then washed the vagina well out with some warm water, by means of a large syringe, which afforded her relief. She appeared to feel excruciating pain while the thick, ropy, latter part of the discharge was coming off. On examination afterwards with the finger, the vagina appeared quite natural, though much contracted, and at the upper part the os tince conld be felt. The large tumour which occupied the iliac and umbilical regions, had completely vaJanuary, $183 \%$.

\section{OBSERVATIONS}

ON MR. ENSOR'S SUPPOSED CASE OF

CHOLERA;

AND THE TREATMENT OF THAT DISEASE WITE BARK.

\section{To the Editor of THE LANCET.}

$\mathrm{S}_{\mathrm{IR}}$, - In your last Number, Jan. 28, a letter from Mr. Ensor, of Portman Square, has been inserted, in which the history of a suspected case of cholera, which occurred at Kuightsbridge on the first of the last montb, is shortly detailed. Among other interesting points in this case, one seems to me especially deserving of attention. The patient was attacked, for the first time, on Sunday, with diarrhœa, romiting, dejected countenance, and lividity of the lips. During the Monday all these symptoms had disappeared, and he "felt himself decidedly improved." On the Tuesday, cold and shivering, pain of epigastric, and considerable exhaustion, were complained of; and again, on Wednesday, he felt so far recovered as to appear " fust approaching a state of convalescence;" when, towards four o'clock in the afternoon, his disease recurred in a more violent form than ever, and he expired on the morning of Thursday.

It cannot escape observation that the vicissitudes which this patient's symptoms sustained throughout his rapid illness, wore a regularly tertian form; Sunday, Tuesday, and T'sursday, being the pyrexial days, wilh anticipating paroxysms; and as this is the promiuent and characteristic feature of the febris perniciosa cholerica of Torti, which some weeks ago I published a brief account of in a letter addressed to Dr. Barry, I have every reason for believing that Mr. Ensor's patient died of this, or a very similar affection.

In my letter to that member of the Central Board of Health, I took occasion to urge the value of rinchona in such diseases; and although it cannot be expected that I should speak positively in a case which I know only imperfectly and by report, I am inclined strongly to believe that had $\mathrm{Mr}$. Ensor substituted bark foi his opium and 